\title{
Atrial Septal Defect Sizing and Transcatheter Closure
}

\author{
Konstantinos Dean Boudoulas ${ }^{a}$ Konstantinos Marmagkiolis ${ }^{b}$ \\ Harisios Boudoulas ${ }^{\mathrm{a}, \mathrm{c}}$ \\ aDivision of Cardiovascular Medicine, Department of Medicine, The Ohio State University, Columbus, OH, USA; \\ ${ }^{b}$ Florida Hospital, Pepin Heart Institute, Tampa, FL, USA; ' ${ }^{B}$ Biomedical Research Foundation, Academy of Athens, \\ Athens, Greece
}

A few decades ago, surgery was the only approach used to correct even simpler congenital (e.g., atrial septal defect, patent ductus arteriosus) or acquired (e.g., mitral stenosis) cardiac abnormalities. Over the last several years, however, due to medical progress and remarkable developments in medical technology, several structural heart diseases (SHD), such as ostium secundum atrial septal defect (ASD), ventricular septal defect, patent foramen ovale, patent ductus arteriosus, mitral stenosis, mitral regurgitation, aortic stenosis, among others, can now be treated in the cardiac catheterization laboratory. ASD was one of the first to be corrected using a transcatheter approach. In fact, today, this method is considered the standard approach in these patients $[1,2]$.

\section{Sizing and Transcatheter Closure of Secundum ASD}

Transcatheter closure of a secundum ASD has been used for years with good results in both children and adults. Precise sizing of the defect is of critical importance for the selection of the appropriate device size. Traditionally, the balloon sizing technique with the guidance of

\section{KARGER}

() 2019 S. Karger AG, Basel

E-Mail karger@karger.com

www.karger.com/crd transesophageal echocardiography (TEE) has been used; however, over the last several years intracardiac echocardiography (ICE) has been increasingly used in place of TEE [3] (Fig. 1). Long-term results (up to this point) using ICE alone to determine ASD size for device selection were not available and for this reason, among others, ICE has not yet fully replaced balloon sizing.

In this issue of Cardiology, Rigatelli et al. [4] present their experience of 281 adult patients undergoing transcatheter closure of an ASD using ICE alone to size the ASD defect. The authors present short- and long-term results with an average of $10.0 \pm 3.0$ years (range 1-15 years). The Amplatzer ${ }^{\mathrm{TM}}$ Septal Occluder (Abbott, St. Paul, MN, USA) was used in 251 patients (mean size 26.4 $\pm 10.2 \mathrm{~mm}$ ), while the Gore ${ }^{\circledR}$ Cardioform Septal Occluder (W. L. Gore and Associates, Inc., Flagstaff, AZ, USA) was used in 30 patients (25-mm device in 21 patients and 30 -mm device in 9 patients). During the follow-up period, a TEE was performed at 1 month and repeated again at 6 months post-closure if there was greater than a trivial shunt seen (12 patients) with the first TEE. Transthoracic echocardiogram was performed at 6 months, at 12 months, and then every 2 years. The procedure was suc- 

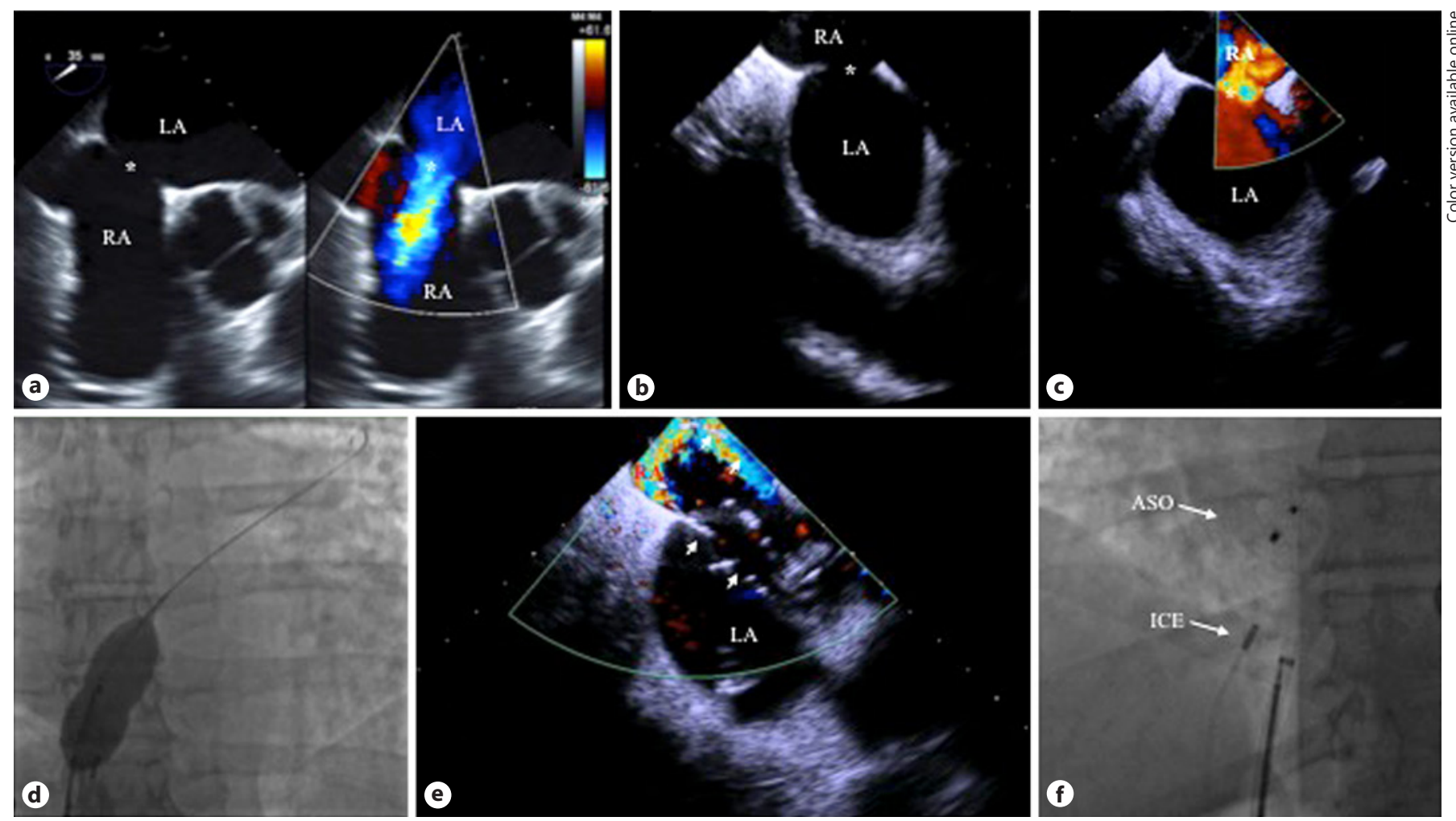

Fig. 1. a TEE with and without color flow Doppler performed during diagnostic evaluation revealed an ostium secundum atrial septal defect (ASD; asterisks) with shunting from the LA to RA. Intraprocedural ICE positioned in the RA without (b) and with (c) color flow Doppler demonstrated an ASD (asterisks). ASD size was determined by balloon (arrows) sizing guided by fluoroscopy (d)

cessful in all patients. Complications related to the procedure were minimal (16 patients: 8 with a groin hematoma, 5 with a pericardial effusion, 2 with temporary atrioventricular block, and 1 with a coronary air embolism). There were no intra-procedural deaths or during the follow-up period. Only 1 patient required surgical removal of an Amplatzer Setpal Occluder due to misalignment that was detected during follow-up TEE that was not seen with ICE during the procedure. On follow-up, compared to pre-procedural values, the mean pulmonary artery pressure decreased from $39.0 \pm 11.3$ to $19.9 \pm 8.1 \mathrm{~mm} \mathrm{Hg}$ $(p<0.01)$, and the mean $\mathrm{Qp} / \mathrm{Qs}$ decreased from $2.4 \pm 0.8$ to $1.1 \pm 0.2(p<0.01)$. Pre-discharge ASD occlusion was achieved in $93.9 \%$ of patients and was observed in $97.8 \%$ of the patients ( 5 moderate shunts and 1 large shunt with device removal) on follow-up. No aortic or atrial wall erosions, or device thrombus were detected during the follow-up period. The authors concluded that ICE-guided ASD closure without the use of balloon sizing was effec- and ICE (e). The balloon was inflated just to the point where there was absent color flow across the ASD as seen by ICE (e). f Deployed Amplatzer Septal Occluder seen on fluoroscopy. LA, left atrium; RA, right atrium; ICE, intracardiac echocardiogram; ASO, Amplatzer Septal Occluder.

tive and safe with very low procedural and late complications. Among other advantages, this approach has been associated with a shorter hospital length-of-stay.

Though balloon sizing is effective, in certain cases, it may overestimate the size of the ASD if the balloon is overstretched, which may lead to device oversizing. Device oversizing may increase the risk of erosion. Further, balloon inflation may produce minor damage to the border of the ASD and may cause chest pain during the procedure, though rare. Though there is a lack of prospective randomized studies to directly compare balloon sizing accompanied with imaging versus using ICE alone in determining ASD size, from available information today, it appears that using ICE alone can be effective and safe. Individualization according to the needs of a patient, physician experience, familiarity with each method, and common sense should be used. When in doubt, balloon sizing should be used if additional information is needed to assist in precisely defining the size of the ASD. 


\section{TEE versus ICE for Transcatheter Closure of ASD}

Regardless of the method used to determine the size of an ASD, TEE or ICE is necessary to guide and monitor catheter and device positioning for appropriate placement of the occluder and to decrease potential complications $[3,5]$. Though TEE is of invaluable importance for the pre-procedure characterization of an ASD, for intraprocedure guidance, ICE offers important advantages and convenience for the operator and the patient. TEE provides superb images, but it requires adequate sedation or general anesthesia due to prolonged esophageal intubation. In addition, esophageal intubation may result in patient discomfort, esophageal damage, or aspiration [5]. On the other hand, ICE can be used with no or minimal sedation. This is an important advantage, especially in the elderly or frail patient $[3,4,6]$. The use of ICE, however, requires venous access that may be associated with vessel damage, thrombosis and/or bleeding/hematoma [6].

Atrial septal anatomy can precisely be defined using ICE and for this reason has been used in the electrophysiology laboratory for years when transseptal procedures were required. Since atrial septal anatomy can be well defined, ICE has been proven to be very effective in guiding transcatheter closures of an ASD and a patent foramen ovale [6,7] (Fig. 1). In a recent nationwide cohort study [3], more than $50 \%$ of ASD closures in adults were performed under ICE guidance, which is the practice at The Ohio State University.

\section{Intra-Procedural Imaging for Other Transcatheter SHD Procedures}

As the number of transcatheter procedures performed for SHD is continuously increasing and the field of SHD is continuously evolving, the need of an imaging modality that does not require extensive sedation or anesthesia for prolonged esophageal intubation is obvious [6]. The use of TEE increases the complexity of case scheduling and resource utilization, as its use typically requires the availability of an anesthesiologist and TEE operator, longer overall procedural times, and extended monitoring post-procedure, which also increases costs. ICE has the potential to become the leading imaging modality in the field of SHD. At present, however, there are certain limitations that prohibit its wide use in transcatheter procedures related to SHD. The two major shortcomings of ICE are its limited viewing depth and inability to provide three-dimensional volumetric imaging [6]. For this reason (with exception of ASD and patent foramen ovale), there are no large studies to confirm the primary role of ICE in transcatheter procedures in the field of SHD [3, 4, 7]. Another potential issue may be the interference of pacemaker leads with the ICE catheter. As technology continues to quickly evolve, new catheters with better imaging and three-dimensional capabilities will soon be available and will overcome these obstacles [8-10]. It is anticipated, therefore, that the use of ICE for most transcatheter procedures in SHD will be a reality in the years to come.

\section{Concluding Remarks}

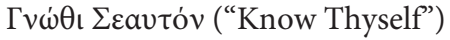

Temple of Apollo at Delphi

There have been great developments in the field of transcatheter procedures related to SHD over the last several years. Clinical scientists and basic investigators have contributed in this area with extensive research. As technology is quickly changing and new technologies are continuously coming into clinical practice, clinicians and interventional cardiologists should use these technologies wisely, efficiently, and effectively. Over the last few decades, we have gradually moved from the operating room to the cardiac catheterization laboratory, and from open chest to closed chest procedures for several cardiac abnormalities. The physician should know what each technology can do and even more importantly what the technology cannot do. Application of new technologies in clinical practice should be based on meticulous research and rigorous clinical assessment. Regardless of progress, each method should be selected because of its usefulness, but consideration also should be given to its hazards, inconvenience, and cost.

\section{Disclosure Statement}

The authors have no conflicts of interest to disclose. 


\section{References}

1 Villablanca PA, Briston DA, Rodés-Cabau J, Briceno DF, Rao G, Aljoudi M, et al. Treatment options for the closure of secundum atrial septal defects: a systematic review and meta-analysis. Int J Cardiol. 2017 Aug;241: $149-55$.

2 Jalal Z, Hascoet S, Baruteau AE, Iriart X, Kreitmann B, Boudjemline $Y$, et al. Long-term complications after transcatheter atrial septal defect closure: A review of the medical literature. Can J Cardiol. 2016 Nov;32(11):1315.e11-8.

3 Alqahtani F, Bhirud A, Aljohani S, Mills J, Kawsara A, Runkana A, et al. Intracardiac versus transesophageal echocardiography to guide transcatheter closure of interatrial communications: nationwide trend and comparative analysis. J Interv Cardiol. 2017 Jun;30(3): 234-41.
4 Rigatelli G, Nghia NT, Zuin, et al. Very longterm outcomes of transcatheter secundum atrial septal defect closure by intracardiac echocardiography without sizing balloon. Cardiology. Forthcoming.

5 Lee AP, Lam YY, Yip GW, Lang RM, Zhang $\mathrm{Q}, \mathrm{Yu}$ CM. Role of real time three-dimensional transesophageal echocardiography in guidance of interventional procedures in cardiology. Heart. 2010 Sep;96(18):1485-93.

6 Alkhouli M, Hijazi ZM, Holmes DR Jr, Rihal CS, Wiegers SE. Intracardial echocardiography in structural heart disease interventions. JACC Cardiovasc Interv. 2018 Nov;11(21): 2133-47.

7 Rigatelli G, Pedon L, Zecchel R, Dell'Avvocata F, Carrozza A, Zennaro M, et al. Long-term outcomes and complications of intracardiac echocardiography-assisted patent foramen ovale closure in 1,000 consecutive patients. J Interv Cardiol. 2016 Oct;29(5):530-8.
8 Friedman TL. Thank you for Being Late. New York: Macmillan Publishers; 2016.

9 Silvestry FE, Kadakia MB, Willhide J, Herrmann HC. Initial experience with a novel real-time three-dimensional intracardiac ultrasound system to guide percutaneous cardiac structural interventions: a phase 1 feasibility study of volume intracardiac echocardiography in the assessment of patients with structural heart disease undergoing percutaneous transcatheter therapy. J Am Soc Echocardiogr. 2014 Sep;27(9):978-83.

10 Pekař M, Kolen AF, Belt $\mathrm{H}$, van Heesch F, Mihajlović N, Hoefer IE, et al. Preclinical testing of frequency tunable capacity micromachined ultrasonic transducer probe prototypes. Ultrasound Med Biol. 2017 Sep;43(9): 2079-85. 